\title{
Knowledge Management and Artificial Intelligence (AI)
}

\author{
Haddy Jallow, Suresh Renukappa, and Subashini Suresh \\ Faculty of Science and Engineering \\ University of Wolverhampton, United Kingdom
}

\begin{abstract}
The fourth industrial revolution known as Industry 4.0 is said to increase the opportunities and industry potential with the use of digitisation aids. The digitisation of the construction industry is becoming very important as processes, quality and efficiency are being focused on a lot more. Within the construction industry, knowledge management (KM) is a key part of learning from past mistakes on previous projects. As part of Industry 4.0, Artificial Intelligence (AI) is one of the technologies that could provide a potential to the construction industry through gathering knowledge from previous projects to determine future project outcomes. Therefore, this research focusses on $\mathrm{AI}$ and its abilities to improve $\mathrm{KM}$ for the construction industry in the UK. Qualitative research approach was adopted to collect and analyse the data. A total of 10 semi-structured interviews were conducted with managers within the construction industry. Results show that organisation have already implemented some sort of AI systems within projects and organisations in order to allow for better KM. Combining AI systems into Common Data Environments can help employees in finding documents easier with a unique ID or referenced words. It is concluded that AI systems can be built and used in order to assist with the KM processes that businesses have already implemented. It is recommended that there is a need for developing a business model canvas of implementing AI to benefit from KM within organisations in order to identify the difference between the business processes without AI for KM and with AI being used to assist KM.
\end{abstract}

Keywords: Artificial Intelligence (AI), Industry 4.0, Digital processes, Knowledge Management, and Construction Industry.

\section{Introduction}

The twenty first century is going through economic changes which are highlighting the importance of Knowledge Management (KM) as value for an organisation. This has been recognised worldwide where knowledge and information are at the forefront of economic growth (Cooke and Leydesdorff, 2006). New patterns of work and business practices have been in development in order to better $\mathrm{KM}$ through technological advancements where the construction industry is referring to this as the fourth industrial revolution. Industry 4.0 includes technological advances such as Artificial Intelligence (AI) which is believed to enable many tasks especially in the managerial role as well as manual workforce to go through simpler processes and have efficient production. A knowledge-based economy tends to be a source of competitiveness for organisations and countries which showcase their creativity and suggests that fresh and new connections are being made and new ways and processes are being formed. In this digital era, there is a need for business establishments and organisational structures to develop innovative and problem-solving processes to encourage the growth of awareness of the digitisation of the construction industry.

This paper aims to identify the key challenges within the construction industry in terms of KM and how AI can be used within the industry to share, capture and use knowledge within and across organisation value chain. This paper is structured in six sections, the research is firstly 
introduced and secondly an in-depth literature review is conducted to gain background information. Methodology is then explained, followed by findings. Thereafter the results are discussed along with the conclusions. This paper contributes to the construction industry where it presents the possible uses of AI within the industry to create better practices and aid business and academia research studies.

\section{Theoretical Background}

\section{Knowledge Management}

Knowledge Management has been a key discipline for over 30 years that explains how knowledge is created, developed, retained and applied within an organisation or country and enables innovation and learning from past knowledge (Soto-Acosta, 2014). KM has been receiving interest for a long time and evidence shows that it is an academic discipline, however it can mature to organisational level where it benefits organisations and workers, as briefly mentioned $\mathrm{KM}$ is now one of the key components to providing a competitive advantage to organisational success. Despite KM being a key component, organisational theory has not fully been developed to provide its significance, in addition, its significance has not been defined to an appropriate universal definition to draw in organisations attention. Knowledge from assets provides organisations a higher understanding of their firms and practice (Nickerson and Zenger, 2004). Gathering knowledge from their assets provides advantages to an organisation such as comparing the gathered knowledge which can aid in the rapid changing environment within the industry.

Similar to the gathering of knowledge from firms, individuals' part of the organisations whom have gained a deep understanding of the organisation's knowledge can also improve their skills leading to the provision of more value towards the organisation. Despite this interpretation being straight forward, pre-existing knowledge is a threat as the ability to learn new knowledge and embrace new processes will be difficult for individuals and organisations to adapt to the change (Tsang and Zahra, 2008). It has been argued that learning of these new processes needs to happen in order to facilitate the new processes of capturing and using pre-exiting knowledge both in an organisational level and an individual level (Suresh et al., 2017, Starbuck, 2001), for this to be possible, the organisational culture needs to change in order to adopt these new knowledge management practices.

\section{Organisational Culture}

Within the construction industry, the biggest difficulty in terms of knowledge is the lack of willingness to share knowledge (Mirzaee and Ghaffari, 2018). There is a lack of understanding in organisation on the cultural benefits of knowledge sharing with better practices and processes. Firms need to be able to be able to identify and assess the nature of their existing knowledge and nurture the knowledge gathered for the sharing of knowledge to be successful and be implemented in a beneficial manner (Koohang, et al., 2008). While there have been a lot of KM research on its successful implementation, there has been a lack of research on what determines a successful KM implementation using AI for an organisation as the cultural implications and management use are highly under-investigated in the construction industry.

\section{Artificial Intelligence and Industry 4.0}


Throughout history, the industries have seen multiple of industrial revolutions which have gradually grown. The Germans introduced the term Industry 4.0 which is aimed at the use of intelligent and digital processes to improve efficiency and productivity within the industry (Jasiulewicz-kaczmarek and Saniuk, 2018). According to Hecklau et al., (2016) the automation process included in the digitisation of the industry which defines Industry 4.0 created more opportunities, however despite this a series of challenges and issues are unleashed which includes issues that have not yet been discovered. Artificial intelligence is the driver of Industry 4.0 where its global market is expected to be worth up to $\$ 15.7$ trillion by 2030 (Microsoft, 2019).

The development of AI to a point at which it matches human capabilities is still a work-inprogress which is debatable if there is a possibility of achieving this. Pratt (1987) argues that knowledge on technology has a gap which needs to be filled in order to understand the engagement between its functionality and reality. AI has been researched on over the past couple of years drawing importance from different sectors, it has captured interest from scientists, automobile sectors to home appliances (Singh, 2018). The definitive goal for AI is to have machines perform tasks to a human being standard if not even better. AI purely focuses on providing knowledge and capabilities, hence without KM, AI processes will be complicated and may be impossible.

AI contains important aspects as stated by Rabuske (1995) in Hoeschl and Barcellos (2006): “... development of heuristical methods for the solution of problems; representation of knowledge; treatment of natural language; acquisition of knowledge; artificial reasoning and logics and tools."

Artificial Intelligence can adopt a variety of definitions, there are two main types of AI which can be distinguished:

- General AI: this refers to a computer system that has the ability to think and act on its own with no supervision, this form of AI currently does not exist.

- Narrow AI: this refers to the form of AI where the computer software is highly sophisticated and uses algorithm techniques to detect patterns with provided data, this form of AI is mainly known as "machine learning” (Broussard, 2018).

In simple definition, AI refers to the introducing of intelligence in machines to provide the capability of performing tasks which would normally require the human mind. AI can be a technique for using data in an efficient manner to allow understanding to the human providing the data (Ghosh, et al., 2018).

In the UK currently, organisations are recognising the benefits of AI which can help in securing the future where it can provide a competitive advantage to the UK's economy. Microsoft's report (2019) highlighted that more than half of organisations in the UK have adopted some sort of AI which shows an increase of $11 \%$ in machine learning, $9 \%$ increase in automation, $7 \%$ rise in voice recognition and an increase of $6 \%$ in smart assistants compares to last year's report (Microsoft, 2019).

According to Fugere (2019), UK organisations full adoption of AI technologies will provide the ability to gain a competitive edge and would allow performance to be enhanced allowing better productivity and profitability.

"The sooner organisations can adopt modern technologies such as AI, the more they can add to the longevity of their business. AI allows you to scale and provide answers." 
Fugere expresses how AI would be beneficial to organisations and states that the application of AI technologies for an organisation can provide many different answers and benefits to the businesses productivity and efficiency for future purposes. Furgere goes on further to state that organisation who adopt AI sooner will gain the most benefits as future savings and efficient processes will be in place while other organisations use old business practices which have been around for many years.

AI systems and development require data to enable an output, similar to KM which data being captured and stored, it can be used to gain knowledge based on future projects and predict outcomes while gaining lessons learnt from previous organisation failures and successes (Antonova et al., 2006).

\section{AI and KM}

There have been a few research cases on both AI and KM, however there is a lack of research on how these can be linked to provide benefits to an organisation. Ilze (2011) has outlined that "since the early 2000s, no survey describing the use of artificial intelligence capabilities in knowledge management tasks has been conducted”. However, Yigitcanlar et al., (2020) through a systematic review of literature noted that AI could improve productivity by automating the data management process and removing the need for intermediaries, and hence increasing profitability. Furthermore, AI can improve the stability and effectiveness of Internet of Things contributing to improved network communication. In return, this would help to improve knowledge sharing, and foster innovation. As there is no clear relation of AI and KM within research, there is a lack of awareness on the capabilities that can be provided with the adoption of AI tools to benefit KM especially in the construction industry. Therefore, this research paper outlines the use of AI and the necessity of KM and how these are both related and can enable each other. Therefore, the research question addressed in this paper is "what are the uses of integrating AI and KM for the benefit of construction organisations"?

\section{Methodology}

This study comprises of semi-structured interview which is a qualitative method. It is a combination of pre-determined customary questions where the interviewee is given the chance to explore a variety of responses, this allows the researcher to gain more of an insight with the responses provided as there are no set answers which limit the input from the interviewees. This form of research is utilised to obtain knowledge and understanding of primary reason, opinions and motivations (Wyse, 2011). This form of research provides an awareness of the issue or assists in providing ideas, uncovering opinions and providing a deeper insight within the problem.

Edmonson and McManus (2007) have provided the reasons as to why in-depth interviews prove to be the right route. Qualitative research provides deep information to be extracted answering the "how", "what" and the "why" of the research questions (Edmonson \& McManus, 2007). Qualitative approach is widely used within the research community to allow complex and evolving phenomena to be studied to be explored within the real-life of organisations or social environments (Yin, 2009).

An idea of an interview guide was obtained through the study of the literature. Interviews were conducted with a total of 10 managers within the construction industry who work in value chain to gain different perspective on KM within their organisation and in addition also gain insight 
on their knowledge on AI and its benefits. The interviews were undertaken through telephone and face to face depending on the location of the interviewees. The interviews spanned from 15 to 30 minutes. These interviews were recorded and then transcribed verbatim. Thematic analysis was adopted for analysis. The interviewees statements were analysed and separated into sub-categories and following this the themes were found.

\section{Results}

Nonaka and Takeuchi (1995) have developed a framework which highlight four key knowledge processes which are socialisation, externalisation, combination and internalisation, AI based KM systems usually support the processes of externalisation, combination and internalisation. Between the years 2004 to 2007, AI in relation to KM was not researched as much as other topics. Over the past three years, KM has become more important for organisations thus creating an interest in intelligent technologies being involved in KM. KM has been further described as personal KM and distributed KM, Figure 1 expands on the different fields and which technologies are used for this type of KM:

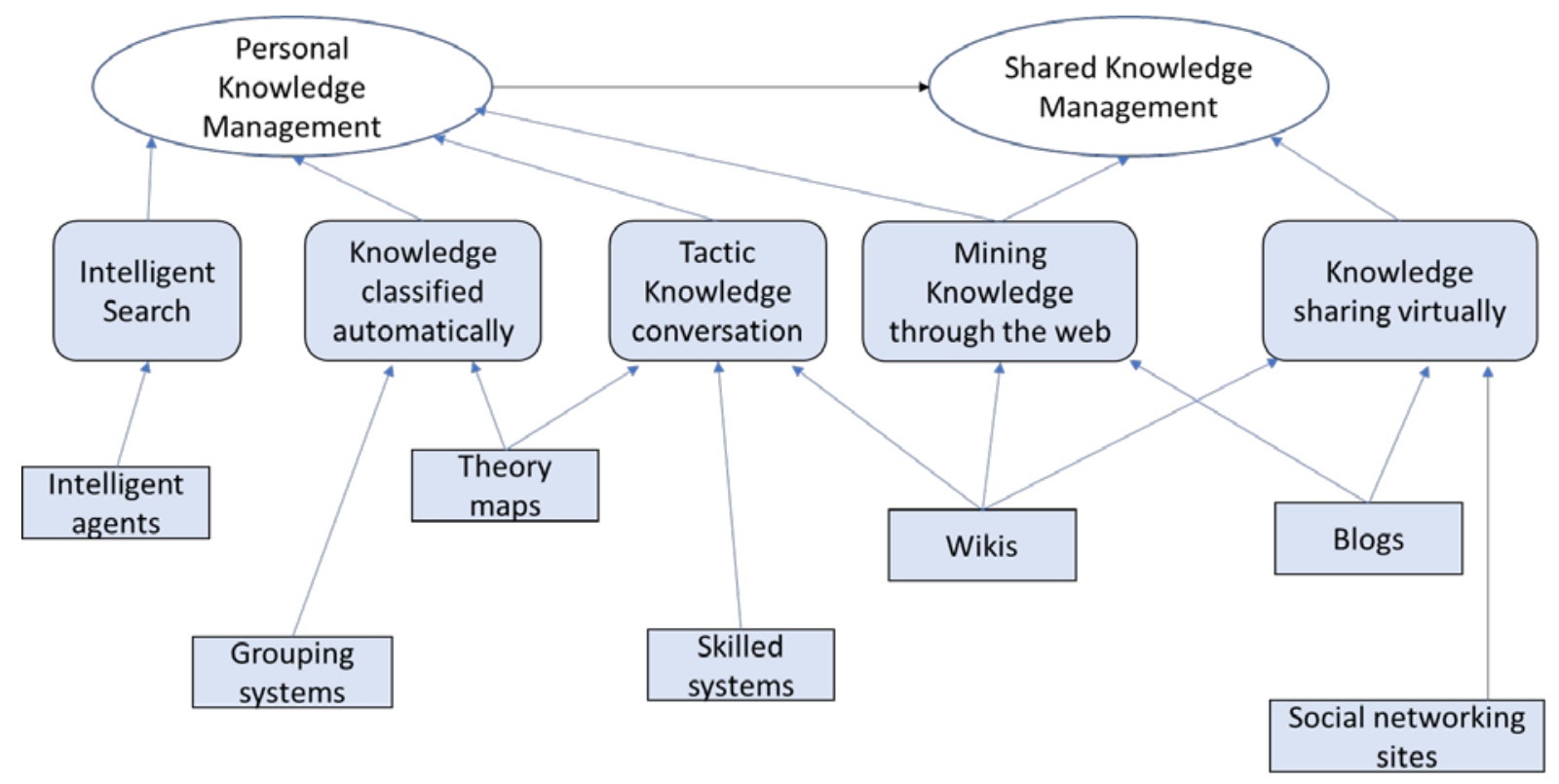

Figure 1: KM fields supported by various AI technologies

\section{Personal KM}

Personal KM is not a new topic it has taken up more of an interest over the past couple of years. The need for access of information is increasing; however the handling of information remains the same (Diao et al., 2009). Computer aids and communications technologies are utilised in order to help persons manage their information in an effective manner. Diao, et al., (2009) have claimed that it is necessary for AI to be incorporated with KM to allow for information to be reliable, quickly derived and accurate.

Artificial Intelligence is one that has not been implemented a lot within the construction industry due to the fact that organisations do not realise its full potential. The interviewees have described the KM practices that are implemented within their organisations and projects. Of the interviewees, $40 \%$ have been using technologies such as Common Data Environments 
(CDEs) to store information within their organisation. For instance, one of the interviewees stated that:

"we have briefed all staff members on going through the process of saving every document whether it be 'work in progress' or 'for construction purposes' into our CDEs so that we can store the data and use it during the handover stage, whilst keeping it in our records for future purposes."

As the teams have been briefed to upload all information within the CDEs which is a form of AI, this allows knowledge to be captured and stored within one environment. This enables knowledge to be used in future for lessons learn purposes and also to share knowledge with future projects. This form of technology has some sort of AI built into it as it recognises the document name and once a new document is uploaded onto the system, it does not allow the same document name used to be repeated as it highlights an error which shows intelligent search.

Of the interviewees, $70 \%$ use a form of automatic classification of knowledge. This is used within the organisation to manage their unstructured data. Software is used to search and detect similarities between documents with built-in coding to create the rules that enable the classification of the data. This enables the organisation to work in an efficient manner in terms of document management.

\section{Distributed KM}

Organisation around the globe are now entering the digital era where work is being transformed virtually. There are increasing demands when it comes to communication and collaboration within organisations which is why information and communication technologies are being included within many different business processes in organisations. The interviewees that took part in this research have implemented forms of AI in order to distribute knowledge within their teams.

Data storage and sharing is all conducted within the same system which recognises people within the organisation and has contact information in addition the documents also contain unique references which allowed the system to use and recognise which document is being searched for.

For instance, $50 \%$ of the interviewees noted that implemented AI technologies such as Power BI into their organisation. Arun Ulag (2018) states

"Power BI makes it possible for every employee in an organization to make better decisions based on data with beautiful reports and dashboards.

With the massive volumes of data generated today about every aspect of a business finding insights from the data can be challenging. This is where AI can help. AI can aid in data exploration, comb through the data to automatically find patterns, help users understand what the data means, and predict future outcomes to help business drive results".

These construction organisations used Power BI as a form of reporting tool to account for the documents contained within the CDE. This allowed the organisation to keep track of documents uploaded, what revision the majority of the documents were, who has logged on to the system and what documents they have downloaded and so on. This form of AI reporting 
tool was useful as it automatically updated itself requiring the minimum human interaction. Knowledge being retained and stored within the AI system was regularly updated allowing the team to gain access to the most up-to-date information while storing their own information.

\section{Discussion}

The construction industry is going through their fourth industrial revolution which is known of as the digital era. Technologies such as building information modelling, virtual reality, unmanned aerial vehicles (Drones) and AI are being used a lot more and incorporated within everyday tasks in the industry to allow for more efficient processes. KM emerged between the early to mid-90's, to enhance productivity and quality as a business strategy. Over the years, $\mathrm{AI}$ and KM researchers have been coming together after decades of research into KM, it was noticed that the two arenas of research compliment each other in terms of creating better processes to enable better KM.

Based on the results obtained through this research, KM has been intensely researched over the years, however there is still a lot to expand on and not one single process can be said is the best process to manage knowledge for an organisation or business. AI has recently been gaining interest within industries over the past couple of years as the construction is entering its fourth industrial revolution which it is being digitalised. Most organisations within the construction industry have been hesitant to implement AI as it would require a large investment at the start, including finding competent staff to being able to interact with the machines however they have also been struggling to adopt an effective way to manage knowledge within their organisations and projects.

Although there is a lack of adoption of both AI and effective KM tools, with the digitalisation of the construction industry there are some AI tools implemented such as the use CDE's which have some machine, human and cloud interaction. This shows that AI is on its way being used as a support for KM within industries, however there can be better uses for AI in terms of KM which can majorly impact and benefit organisations and businesses. Therefore, this paper has explored and reported the use of integrating AI and KM in two ways. They are personnel and distributed knowledge. Both of these are used for the benefit of construction organisations.

\section{Conclusion}

Implementing the right KM strategy is a difficult business decision. The capturing, sharing and transferring of knowledge is a key part of businesses as lessons learnt and best practices can be documented allowing future projects to learn from successes/mistakes and shared with others. Results show that organisation have already implemented some sort of AI systems within projects and organisations in order to allow for better KM. Combining AI systems into Common Data Environments can help employees in finding documents easier with a unique ID or referenced words. The construction industry contains a lot of paperwork and having to go through possibly tens of thousands of documents to find one needed document can be very tedious, however with AI built into the system it provides an efficient route for employees to access information and knowledge faster. It is concluded that AI systems can be built and used to assist with the KM processes that businesses have already implemented. However, AI is one of the fourth industrial revolution applications which have not been fully adopted within the construction industry. Though AI has not been adopted by many organisations due to the investments at start, organisations hesitate as they are not fully aware of its full benefits and how it could boost KM within their teams. Therefore, there is a need for developing a business 
model canvas of implementing AI to benefit KM within organisations in order to identify the difference between the business processes without AI for KM and with AI being used to assist $\mathrm{KM}$. This paper contributes to organisations within the construction industry value chain use personnel and distributed knowledge in terms of the possibilities of using AI for KM within their organisations. To gain more insight and further this research, it would be beneficial to explore the implementation of AI on at least one organisation as a case study to establish the results of barriers and benefits on the use of AI to support KM within that organisation. This will allow rich and deeper insight within this field.

\section{References}

Antonova, A., Gourova, D. E., and Nikolov, D. R. (2006). Review of technology solutions for knowledge management. 2nd IET International Conference on Intelligent Environments, 39-44.

Birzniece, I. (2011). Artificial Intelligence in Knowledge Management: Overview and Trends. DBLP.

Broussard, M. (2018). Artificial Unintelligence How Computers Misunderstand the World. Philadelphia: Harper's.

Cooke, P., and Leydesdorff, L. (2006). Regional Development in the Knowledge-Based Economy: The Construction of Advantage. The Journal of Technology Transfer volume , 5-15.

Cowley-Cunningham, M. B. (2017). Online Business Review of Susskind, R., \& Susskind, D. (2015). The Future of the Professions: How Technology will Transform the Work of Human Experts - Oxford University Press, UK. AB Marketing.

Diao, L., Zuo, M., and Lui, Q. (2009). The Artificial Intelligence in personal Knowledge Management . Second International Symposium on Knowledge Acquisition and Modelling, 327-329.

Edmonson, A. C., and McManus, S. E. (2007). methodological fit in management field research, Academy of Management Review, 1155-1179.

FabianHecklau, MilaGaleitzke, SebastianFlachs, and HolgerKohl. (2016). Holistic Approach for Human Resource Management in Industry 4.0. CIRP, 1-6.

Ghosh, A., Chakraborty, D., and Law, A. (2018). Artificial intelligence in Internet of things. CAAI Transactions on Intelligence Technology, 208-218.

Hoeschl, H. C., and Barcellos, V. (2006) Artificial intelligence, Juridical Intelligence and Systems IJURIS, 11-19.

Jasiulewicz-kaczmarek, M., and Saniuk, A. (2018). How to Make Maintenance Processes More Efficient Using Lean Tools? Advances in Intel-ligent Systems and Computing , 9-20.

Koohang, A., Harman, K., and Britz, J. (2008). Knowledge Management: Theoretical Foundations. California: Informing Science Press.

Microsoft. (2019). Accelerating competitive advantage with AI. Microsoft corporation.

Mirzaee, S., and Ghaffari, A. (2018). Investigating the impact of information systems on knowledge sharing. Journal of Knowledge Management, 501-520.

Nickerson, J. A., and Zenger, T. R. (2004). A Knowledge-Based Theory of the Firm-The ProblemSolving Perspective. Organization Science, 617-632.

Nonaka, I. and Takeuchi, H. (1995) The Knowledge-creating Company: How Japaneses Companies Create the Dynamics of Innovation, Oxford University Press, Oxford. 
Pratt, D. D. (1987). Technology and instructional functions. New Directions for Adult and Continuing Education.

Singh, R. (2018, April 11). The bond between Artificial Intelligence and Knowledge Management. Retrieved from CURATTI: https://curatti.com/artificial-intelligence-knowledge-management/

Soto-Acosta, P. (2014). E-business, organizational innovation and firm performance in manufacturing SMEs: an empirical study in Spain. Technological and Economic Development of Economy , 885-904.

Starbuck, W. H. (2001). How Organizations Learn from Success and failure. Organizational Learning and Knowledge, 327-350.

Suresh, S., Olayinka, R., Chinyio, E., and Renukappa, S., (2017) Impact of knowledge management practices on construction projects, Proceedings of the Institution of Civil Engineers - Management, Procurement and Law, 170(1), 27-43. http://dx.doi.org/10.1680/jmapl.15.00057

Tsang, E. W., and Zahra, S. A. (2008). Organizational unlearning. Sage Journals, 917-931.

Ulag, A. (2018) Microsoft Power BI Blog. Retrieved from Microsoft: https://powerbi.microsoft.com/en-us/blog/power-bi-announces-new-ai-capabilities/

Whittington, R. (2015). Giddens, structuration theory andstrategy as practice. Cambridge Handbook of Strategy as practice, 109-126.

Yigitcanlar, T., Desouza, K.C., Butler, L. and Roozkhosh, F., (2020). Contributions and risks of artificial intelligence (AI) in building smarter cities: Insights from a systematic review of the literature. Energies, 13(6), p.1473.

Yin, R. K. (2009). Case Study Research: Design and Methods, Thousand Oaks, CA: Sage Publications. 